\title{
COMMENTARY
}

\section{Hemodynamic changes during weaning: can we assess and predict cardiac-related weaning failure by transthoracic echocardiography?}

\author{
Gorazd Voga* \\ See related research by Caille et al., http://ccforum.com/content/14/3/R120
}

\begin{abstract}
Cardiac-related failure of weaning from mechanical ventilation is an important reason for prolonged mechanical ventilation, intensive care unit treatment, and increased morbidity and mortality. When transthoracic echocardiography (TTE) is routinely performed before a weaning trial, patients at high risk of cardiac-related failure can be detected by low left ventricular (LV) ejection fraction, diastolic dysfunction, and elevated LV filling pressure. During the weaning trial, a further increase of LV filling pressure and progression of diastolic failure can be observed by repeated TTE. Owing to certain limitations concerning patients and methodology, TTE cannot be employed in every patient and invasive hemodynamic monitoring is still mandatory in selected patients with repetitive weaning failure.
\end{abstract}

In the previous issue of Critical Care, Caille and colleagues [1] evaluated the ability of transthoracic echocardiography (TTE) to predict cardiac-related weaning failure and to assess the hemodynamic changes before and 30 minutes after the start of a spontaneous breathing trial (SBT). The authors measured maximal velocities of mitral $\mathrm{E}$ and $\mathrm{A}$ waves, deceleration time of $\mathrm{E}$ wave (DTE), maximal velocity of E' wave measured by tissue Doppler at lateral mitral annulus, and left ventricular (LV) stroke volume.

Weaning failed in 23 of 117 patients, and failure was of cardiac origin in the majority (20 of 23 ) of them. In patients with weaning failure, a significantly lower heart

*Correspondence: gorazd.voga@guest.arnes.si

Medical Intensive Care Unit, General Hospital Celje, Oblakova 5, 3000 Celje, Slovenia

BioMed Central o 2010 Biomed Central Ltd rate and $E / E^{\prime}$ ratio and a higher left ventricular ejection fraction (LVEF) were observed before SBT. During SBT, significant increases in cardiac output, systolic arterial pressure, and E/A relation and a nonsignificant increase in $E / E$ ' were observed, with significant shortening of DTE. Weaning failure was observed in $17 \%$ of patients with LVEF of greater than 50\%, $13 \%$ of patients with LVEF of $35 \%$ to $50 \%$, and $31 \%$ of patients with LVEF of less than $35 \%$. Before SBT, DTE was significantly shorter and E/E' was significantly higher in patients with LVEF of less than 35\%. During SBT, E/A increased and DTE decreased significantly in patients with LVEF of less than $50 \%$.

If these results are translated into simple clinical language, patients with weaning failure were tachycardic and had depressed systolic function and diastolic dysfunction (short DTE) with elevated LV filling pressure (high E/E' ratio) before SBT. During SBT, a further increase in LV filling pressure (increased $\mathrm{E} / \mathrm{A}$ and $\mathrm{E} / \mathrm{E}^{\prime}$ ratio) and deterioration of diastolic function were observed.

TTE was therefore able to identify patients who were at risk of cardiac-related weaning failure by measuring relatively simple and reproducible variables (LVEF, E/E,' and DTE) before SBT. Moreover, hemodynamic changes (increase of pulmonary artery occlusion pressure [PAOP] and changes in stroke volume) during SBT can be followed by repeated TTE.

Unsuccessful weaning from mechanical ventilation occurs in approximately $20 \%$ to $30 \%$ of patients and is related to prolonged mechanical ventilation, length of stay in the intensive care unit, and increased morbidity and mortality [2]. It is more frequent in patients with chronic obstructive lung disease or pre-existing heart disease or both. Cardiac failure is usually due to cardiogenic pulmonary edema or to inadequate response to the increased oxygen demand [3,4]. Hemodynamic monitoring by pulmonary artery catheter (PAC) is traditionally used in patients with unsuccessful weaning for monitoring PAOP, cardiac output, and mixed venous oxygen saturation and enables proper diagnosis and treatment 
with vasodilators, diuretics, and inodilators. Since invasive hemodynamic monitoring is less frequently used, noninvasive alternatives for assessment of cardiacrelated weaning failure such as measurement of baseline brain natriuretic peptide values and its increase during weaning, hemoconcentration during SBT, and echocardiographic examination before and during weaning have been studied [5-7]. Echocardiography allows the assessment of systolic and diastolic cardiac function and etiologic diagnosis of cardiac disease. Through the analysis of transmitral flow and tissue Doppler velocities, it is also possible to estimate the LV filling pressure change during weaning [8].

Concerning the results of the study, two clinically important questions should be answered. First, should we routinely use TTE in patients before weaning? If we take into account the ability of TTE to predict difficult weaning, the answer is undoubtedly yes. The problem is the fact that an experienced echocardiographist is required for performing and interpreting the TTE in such patients [9]. Moreover, TTE cannot be performed in all patients, and patients with inadequate visibility and image acquisition, atrial fibrillation, and paced rhythm must be excluded [1]. Despite these drawbacks, echocardiography is increasingly used in the majority of intensive care units as the most common available noninvasive method for objective hemodynamic assessment. Therefore, it should be routinely used before SBT for hemodynamic assessment and estimation of the risk of cardiac-related weaning problems.

The second question is whether we can detect and monitor the hemodynamic changes during SBT accurately enough to titrate the treatment of cardiac failure. The answer is probably no. It is technically impossible and absolutely too time-consuming to perform TTE every few minutes during SBT. Besides, the changes of specific variables measured by TTE are relatively small and cannot be used for treatment adjustment.

In real life, the practical approach should include TTE before SBT and treatment in patients who are at high risk of weaning failure. In the case of cardiac-related weaning failure, the second SBT should probably be performed with close hemodynamic monitoring by PAC.

\section{Abbreviations}

DTE, deceleration time of E wave; LV, left ventricular; LVEF, left ventricular ejection fraction; PAC, pulmonary artery catheter; PAOP, pulmonary artery occlusion pressure; SBT, spontaneous breathing trial; TTE, transthoracic echocardiography.

Competing interests

The author declares that he has no competing interests.

Published: 7 July 2010

References

1. Caille V, Amiel JB, Charron C, Belliard G, Vieillard-Baron A, Vignon P. Echocardiography: a help in the weaning process? Crit Care 2010, 14:R120.

2. Ely EW, Baker AM, Dunagan DP, Burke HL, Smith AC, Kelly PT, Johnson MM, Browder RW, Bowton DL, Haponik EF: Effect on the duration of mechanical ventilation of identifying patients capable of breathing spontaneously. NEngl J Med 1996, 335:1864-1869.

3. Lemaire F, Teboul JL, Cinotti L, Giotto G, Abrouk F, Steg G, Macquin-Mavier I, Zapol WM: Acute left ventricular dysfunction during unsuccessful weaning from mechanical ventilation. Anesthesiology 1988, 69:171-179.

4. Jubran A, Mathru M, Dries D, Tobin MJ: Continuous recordings of mixed venous oxygen saturation during weaning from mechanical ventilation and the ramifications thereof. Am J Respir Crit Care Med 1998, 158:1763-1769.

5. Mekontso-Dessap A, de Prost N, Girou E, Braconnier F, Lemaire F, Brun-Buisson C, Brochard L: B-type natriuretic peptide and weaning from mechanical ventilation. Intensive Care Med 2006, 32:1529-1536.

6. Grasso S, Leone A, De Michele M, Anaclerio R, Cafarelli A, Ancona G, Stripoli T, Bruno F, Pugliese P, Dambrosio M, Dalfino L, Di Serio F, Fiore T: Use of $\mathrm{N}$-terminal pro-brain natriuretic peptide to detect acute cardiac dysfunction during weaning failure in difficult-to-wean patients with chronic obstructive pulmonary disease. Crit Care Med 2007, 35:96-105.

7. Anguel N, Monnet X, Osman D, Castelain V, Richard C, Teboul JL: Increase in plasma protein concentration for diagnosing weaning-induced pulmonary oedema. Intensive Care Med 2008, 34:1231-1238.

8. Lamia B, Maizel J, Ochagavia A, Chemla D, Osman D, Richard C, Teboul JL: Echocardiographic diagnosis of pulmonary artery occlusion pressure elevation during weaning from mechanical ventilation. Crit Care Med 2009, 37:1696-1701

9. Price S, Via G, Sloth E, Guarracino F, Breitkreutz R, Catena E, Talmor D and World Interactive Network Focused On Critical UltraSound ECHO-ICU Group: Echocardiography practice, training and accreditation in the intensive care: document for the World Interactive Network Focused on Critical Ultrasound (WINFOCUS). Cardiovasc Ultrasound 2008, 6:49.

doi:10.1186/cc9085

Cite this article as: Voga G: Hemodynamic changes during weaning: can we assess and predict cardiac-related weaning failure by transthoracic echocardiography? Critical Care 2010, 14:174. 